\title{
Supplementary Information for " Photoinduced and Thermal Relaxation in Surface-grafted Azobenzene-based Monolayers: A Molecular Dynamics Simulation Study."
}

\author{
Dmitry Bedrov", Justin B. Hooper , Mathew A. Glaser", and Noel A. Clark ${ }^{\#}$ \\ "Department of Materials Science \& Engineering, University of Utah. \\ "Department of Physics, University of Colorado at Boulder.
}

\section{Force field.}

In order to find higher energy conformers, we have constrained each torsion independently to generate a full 360-degree torsional rotation in 10 degree increments, with all other torsional degrees of freedom allowed to relax. This allowed us to extract geometries representing potentially stable conformations, from which we performed full molecular geometry optimizations to recover the nearest stable state. Through this approach, we have identified three local minima via quantum chemistry (QC) calculations, which are presented in Figure SI-1. The characteristic dihedrals are labeled on the figure, where all dihedrals terminate on the oxygen and a carbon atom, with the termination carbon of $\phi_{2}$ chosen as the one bonded to the Azo bridge between aromatic rings.

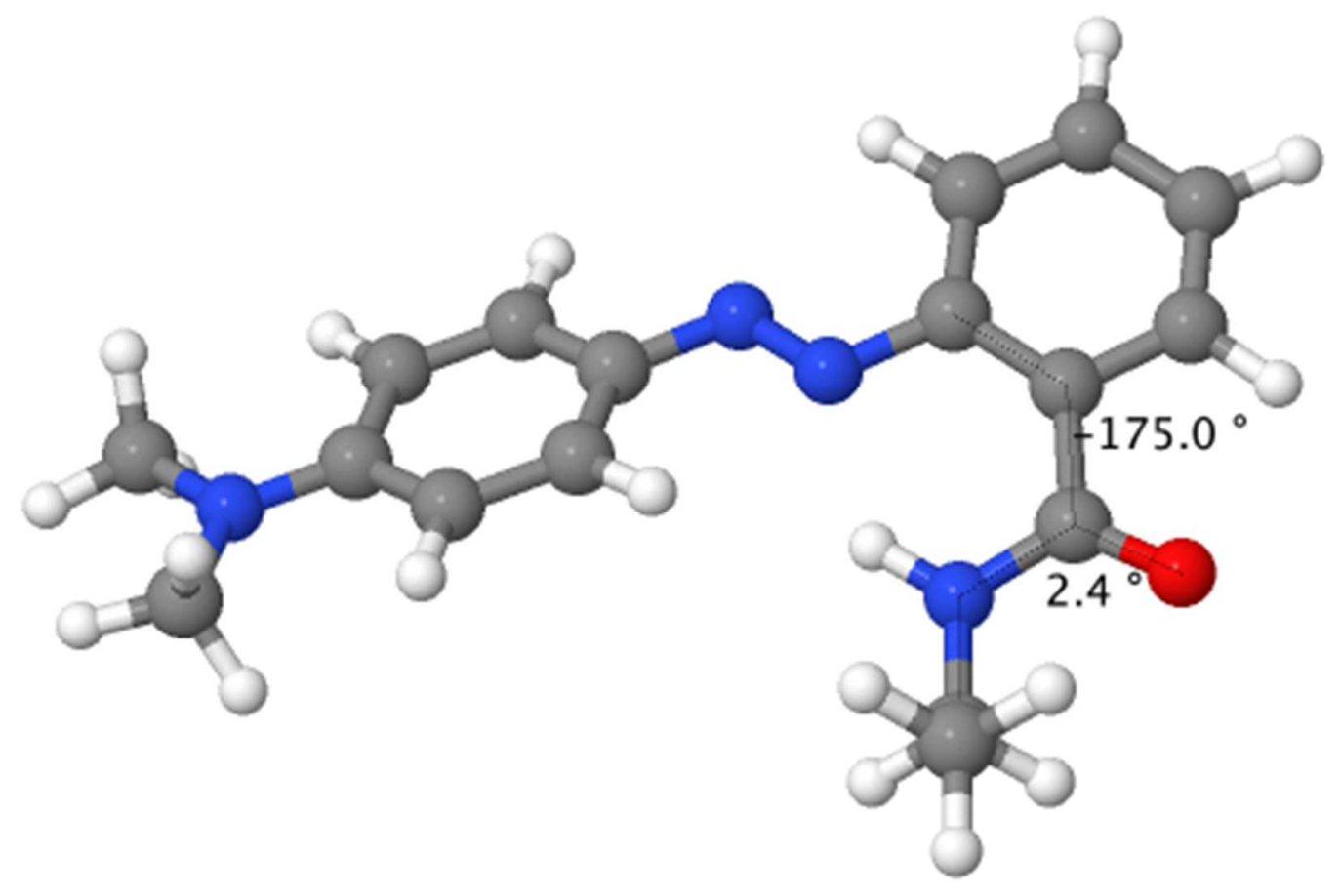

Figure SI-1

M052X//cc-pvDz minimum energy configuration of the Azobenzene species used to parameterize the APPLE\&P forcefield. The top number represents the measured dihedral angle of the CCCO dihedral, while the bottom represents the measured angle of the OCNC dihedral. 




Figure SI-2

M052X//cc-pvDz configuration of the Azobenzene species used to parameterize the APPLE\&P forcefield. The top number represents the measured dihedral angle of the CCCO dihedral, while the bottom represents the measured angle of the OCNC dihedral. The overall energy of this conformation was predicted to be 5.6 $\mathrm{kCal} / \mathrm{mol}$ via DFT, and $5.3 \mathrm{kCal} / \mathrm{mol}$ higher via APPLE\&P.

The minimum energy state is the one presented in Fig. SI-1a (and schematically represented in 1a), while QC calculations predict the next lowest minimum (Figure SI-2) occurs when $\phi_{3}$ rotates through approximately 55 degrees. The energy penalty of this configuration is roughly $5.6 \mathrm{kcal} / \mathrm{mol}$, presumably representing the cost of breaking the amine/azo hydrogen bond. Addition of roughly another $1 \mathrm{kcal} / \mathrm{mol}$ to the molecule allows the azobenzene group to cooperatively reorient to a more planar, rather than upright configuration, which involves reorientation of both $\phi_{2}$ and $\phi_{3}$ as demonstrated in Figure SI-3. 


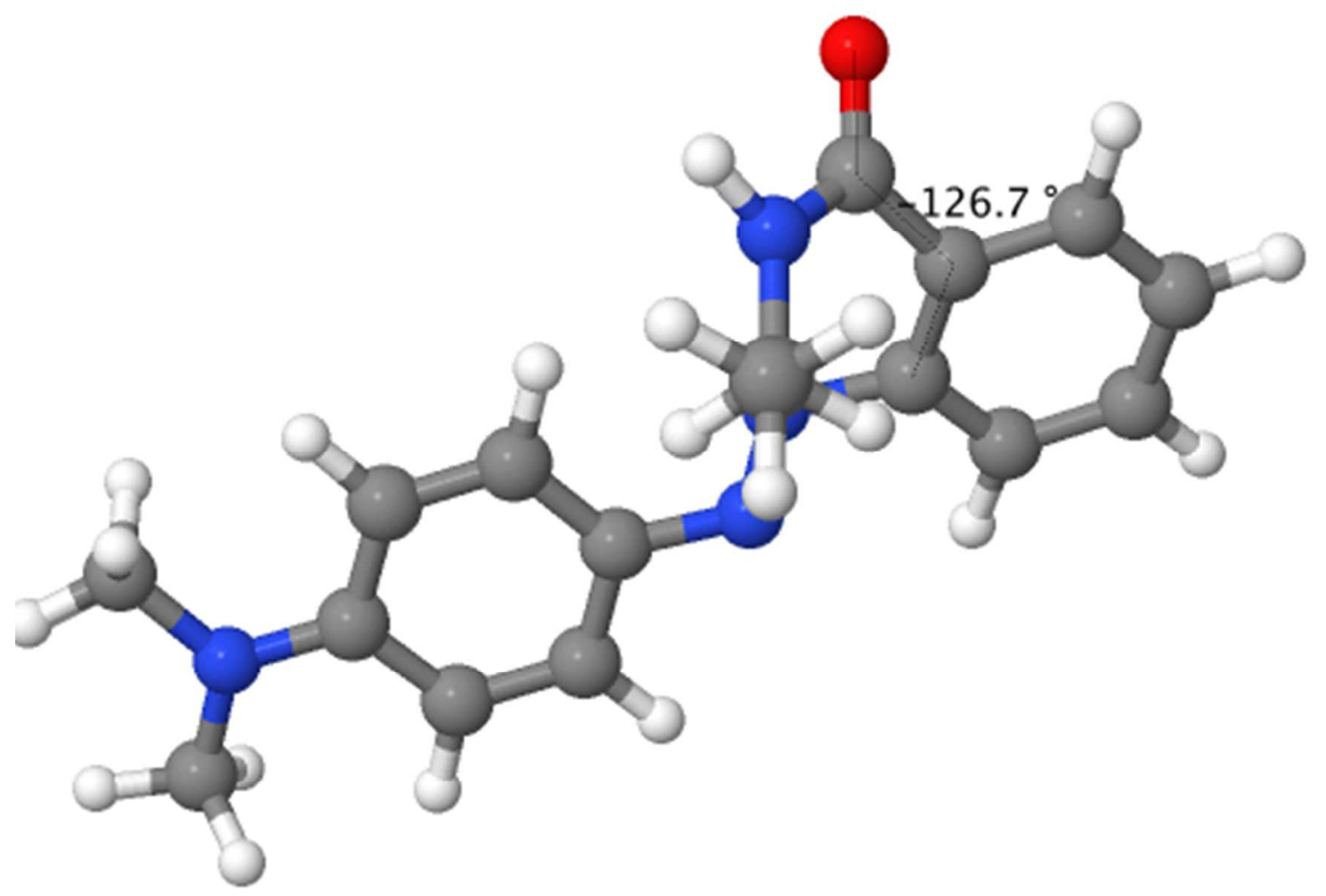

Figure SI-3a

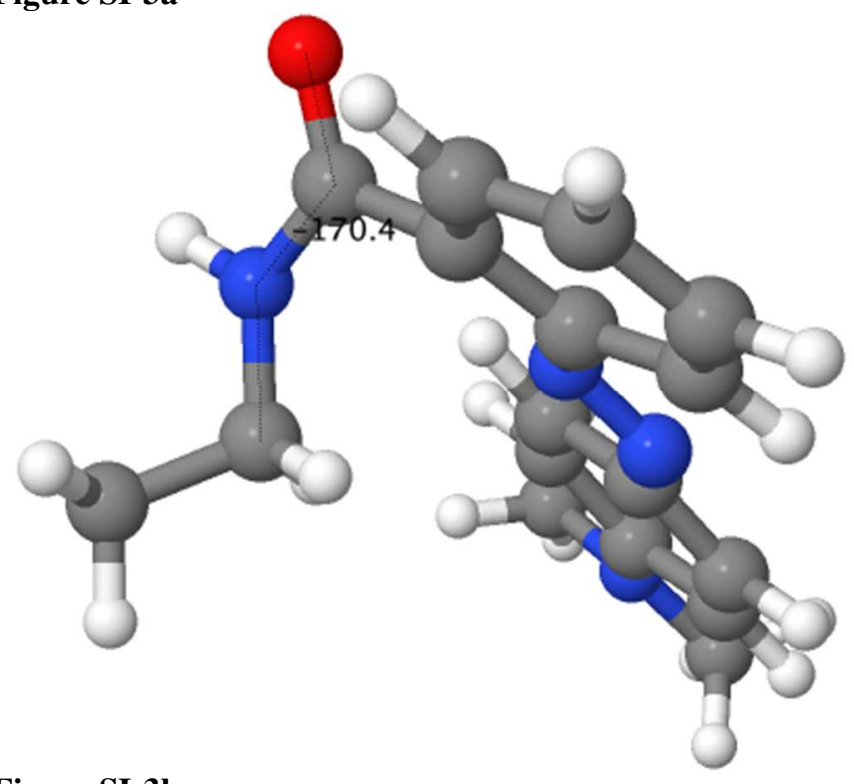

Figure SI-3b

Local minimum energy state with relative energy offset of $6.6 \mathrm{kCal} / \mathrm{mol}$ compared to the global minimum of Figure SI-1. For ease of visualization, Figure SI-3a dhows the value of the CCCO dihedral, while (b) shows the value of the OCNC dihedral, as well as presenting a sense of the extent to which the azobenzene group has oriented relative to the surface on which the SAM is deposited.

APPLE\&P generates similar geometrical configurations, but predicts that the configuration in Fig. SI-3 is roughly $1 \mathrm{kcal} / \mathrm{mol}$ lower in energy, rather than higher in 
energy, compared to the configuration of Fig. SI-2. APPLE\&P still predicts the low energy conformation in line with that seen in QC calculations, although the value of $\phi_{3}$ is shifted by $\sim 16$ degrees, due to steric interactions between the amine hydrogen and the azo nitrogen. A similar angular increase is also seen in Fig. SI-2, also likely due to steric interactions in the FF, this time between the azo nitrogen and the oxygen. This data is summarized in Table SI-1.

Table SI-1

Energy and angles of local minima of dMR molecule tether torsional angles

\begin{tabular}{|l|c|c|c|c|c|c|}
\hline & \multicolumn{2}{|c|}{ QC (M052X//cc-pvDz) } & \multicolumn{3}{c|}{ APPLE\&P } \\
\hline & $\Delta \mathrm{E}(\mathrm{kcal} / \mathrm{mol})$ & $\phi_{2}$ & $\phi_{3}$ & $\Delta \mathrm{E}(\mathrm{kcal} / \mathrm{mol})$ & $\phi_{2}$ & $\phi_{3}$ \\
\hline Minimum (Fig. 1a) & 0.0 & $-175^{\circ}$ & $2^{\circ}$ & 0.0 & $-159^{\circ}$ & $6^{\circ}$ \\
\hline Perpendicular & 5.6 & $50^{\circ}$ & $-1.7^{\circ}$ & 5.3 & $63^{\circ}$ & $5.6^{\circ}$ \\
\hline Parallel & 6.3 & $-127^{\circ}$ & $-170^{\circ}$ & 4.3 & $-131^{\circ}$ & $-169^{\circ}$ \\
\hline
\end{tabular}

\section{System set up.}

A monolayer system comprised of 256 molecules was prepared in the following way. Initially, a square surface with side length $L=37 \mathrm{~nm}$ (corresponding to a surface area per molecule of $5.3 \mathrm{~nm}^{2} /$ molecule) has been divided into 16x16 square lattice. Each molecule was placed and attached to the surface within one lattice cell as shown in Figure SI-4 bellow. The location of the attachment point for each molecule within the cell $\left(x_{\mathrm{i}}, y_{\mathrm{i}}\right)$ was randomly picked subject to no-overlap condition with the molecules from neighboring cells. All molecules were initially in trans conformation of the azobenzene unit and had a random orientation in the $\mathrm{x}-\mathrm{y}$ plane. Subsequently, a simulation run over $1 \mathrm{~ns}$ was used to shrink the $\mathrm{x}$ and $\mathrm{y}$ dimensions (directions parallel to the surface) of the surface to $12.4 \times 12.4 \mathrm{~nm}^{2}$ dimensions allowing the desired surface coverage of $0.60 \mathrm{~nm}^{2} / \mathrm{mol}$ (see Figure SI-4. Green points on the figure illustrate the attachment points of dMR molecules to the surface. During this simulation the attachment points did not change their scaled coordinates, i.e. $x_{i} / L$ and $y_{i} / L$ ratios remained constant. This initial set up simulation was conducted at $298 \mathrm{~K}$. A configuration of the prepared monolayer is shown in Figure $1 \mathrm{~b}$. 
Subsequently, a 5 ns equilibration run was conducted in the NVT ensemble for each system after its preparation.

Initial set up configuration 256 molecules on $37 \times 37 \mathbf{n m}^{2}$ surface

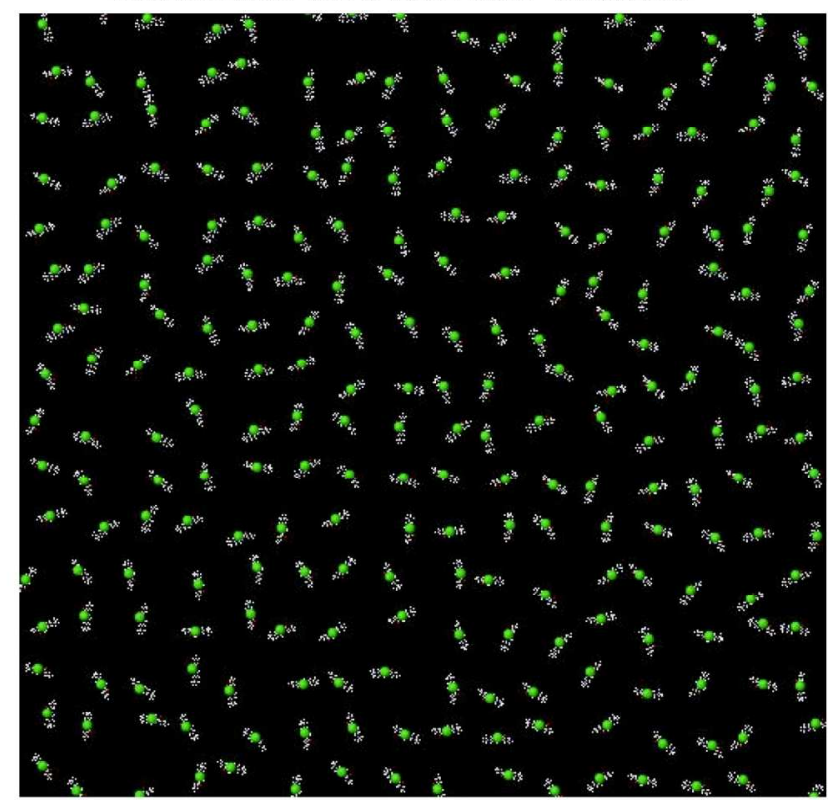

Final (after shrinking) set up configuration 256 molecules on $12 \times 12 \mathrm{~nm}^{2}$ surface

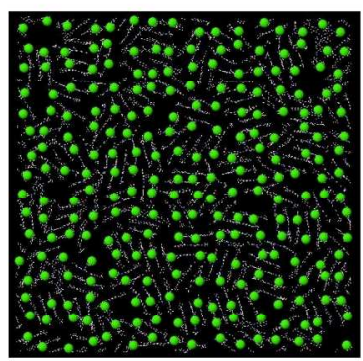

Green atoms show attachment points to the surface for each dMR molecule.

\section{Figure SI-4}

Snapshots of initial set up configuration (grafting density $5.34 \mathrm{~nm}^{2} /$ molecule) and final configuration (grafting density $0.6 \mathrm{~nm}^{2} /$ molecule). Green atoms indicate anchoring points to the surface for each $\mathrm{dMR}$ molecule. 